\title{
New Liouville theorems for linear second order degenerate elliptic equations in divergence form ${ }^{\text {th }}$
}

\section{Nouveaux théorèmes de Liouville pour des équations elliptiques dégénérés linéaires du second ordre en forme de divergence}

\author{
Luisa Moschini \\ Dipartimento di Matematica “G. Castelnuovo”, Università di Roma "La Sapienza”, P.le A. Moro 5, 00185 Roma, Italy \\ Received 25 November 2003; accepted 23 March 2004 \\ Available online 29 July 2004
}

\begin{abstract}
In this paper we give conditions on the positive function $\varphi^{2}$ under which every bounded solution $\sigma$ of the elliptic equation $\nabla \cdot\left(\varphi^{2} \nabla \sigma\right)=0$ in $\mathbb{R}^{n}$ must be constant. The case when $\varphi^{2}$ only depends on one or two variables is discussed at length. Moreover the asymptotic behavior of possibly unbounded solutions is characterized, improving in such a way a Liouville theorem due to Berestycki, Caffarelli and Nirenberg.

(C) 2005 L'Association Publications de l'Institut Henri Poincaré. Published by Elsevier B.V. All rights reserved
\end{abstract}

\section{Résumé}

Dans ce papier nous donnons des conditions sur la fonction positive $\varphi^{2}$ sous lesquelles toute solution de l'équation elliptique $\nabla \cdot\left(\varphi^{2} \nabla \sigma\right)=0$ en $\mathbb{R}^{n}$ doit être constante. Le cas où $\varphi^{2}$ ne dépend que d'une ou deux variables est analysé en détail. Ensuite, le comportement asymptotique des solutions, éventuellement non bornées est characterisé, en donnant ainsi une généralisation d'un théorème de Liouville dû à Berestycki, Caffarelli et Nirenberg.

(C) 2005 L'Association Publications de l'Institut Henri Poincaré. Published by Elsevier B.V. All rights reserved

MSC: primary 35J70; secondary 35B50

Keywords: Linear degenerate elliptic equation; Liouville-type theorem; Maximum principle

\footnotetext{
Work partially supported through RTN Contract HPRN-CT-2002-00274.

E-mail address: moschini@mat.uniroma1.it (L. Moschini).
} 


\section{Introduction}

We shall be concerned here with the elliptic equation $\nabla \cdot\left(\varphi^{2} \nabla \sigma\right)=0$ on the Euclidean space $\mathbb{R}^{n}$; the weight function $\varphi^{2}$ is chosen to be $\varphi^{2}(x)>0, x=\left(x_{1}, \ldots, x_{n}\right) \in \mathbb{R}^{n}, n \geqslant 1$.

The symbol $\nabla \sigma$ denotes the gradient vector $\left(\frac{\partial \sigma}{\partial_{x_{1}}}, \ldots, \frac{\partial \sigma}{\partial_{x_{n}}}\right)$ of the solution $\sigma=\sigma(x)$, and $\nabla \cdot\left(\varphi^{2} \nabla \sigma\right)$ stands for $\sum_{i=1}^{n} \frac{\partial}{\partial x_{i}}\left(\varphi^{2} \frac{\partial \sigma}{\partial x_{i}}\right)$.

As usual we will say that the equation is uniformly elliptic if the function $\varphi^{2}$ is strictly positive and bounded, that is $0<C \leqslant \varphi^{2} \leqslant C^{-1}$, for some constant $C$; on the other end the case in which $\varphi^{2}$ decays to zero at infinity will be referred to as degenerate elliptic.

Liouville-type results will be understood to mean, as usual, assertions to the effect that the only bounded solutions $\sigma=\sigma(x)$ of the equation $\nabla \cdot\left(\varphi^{2} \nabla \sigma\right)=0$ in $\mathbb{R}^{n}$ are the constants.

Here we prove new Liouville theorems which are motivated by a famous symmetry question raised by E. De Giorgi. It concerns the one-dimensional character of bounded monotone solutions of semilinear elliptic problems in the whole $n$-dimensional space.

The main purpose of this paper is to establish conditions on the weight $\varphi^{2}$ under which a Liouville theorem holds.

For instance in the uniformly elliptic case a Liouville theorem is known to hold on $\mathbb{R}^{n}$ for any $n$ (De GiorgiNash-Moser, see [7,17,18]).

Things considerably change when one does not assume uniform ellipticity.

When $n=2$ a Liouville-type result is known to apply to general (positive and) bounded weights $\varphi^{2}$ (see [9,11]). On the other hand in higher dimensions, $n \geqslant 3$, a counterexample, obtained in [2] by means of a probabilistic approach, shows that a Liouville theorem does not a priori holds true under the sole boundedness assumption on the positive function $\varphi^{2}$.

Thus when $n \geqslant 3$ a Liouville theorem may hold true only under some additional assumptions on the weight function $\varphi$. In this direction in [16] a Liouville theorem is given under certain integrability conditions on $\varphi^{2}$ (see $[22,8])$; more recently by means of a probabilistic approach a powerful result is given in [3]. Another direction which has been widely investigated is the one of radial weight functions, thus $\varphi(x):=\varphi(\|x\|)($ see $[11,14,13]$ among others). Indeed when $\varphi$ and $\sigma$ are sufficiently smooth, the equation under investigation takes the following form: $\Delta \sigma+\left\langle\frac{2 \nabla \varphi}{\varphi}, \nabla \sigma\right\rangle=0$, where $\langle$,$\rangle denotes the standard scalar product in \mathbb{R}^{n}$. A Liouville-type theorem is then known to apply when "lower order terms" $\frac{2 \nabla \varphi}{\varphi}$, vanish at a sufficiently fast rate as $\|x\| \rightarrow \infty$, more precisely when $\frac{2 \nabla \varphi}{\varphi}=\mathrm{O}\left(\|x\|^{-1}\right)$ (see [11]). The $\mathrm{O}\left(\|x\|^{-1}\right)$-decay condition, which cannot be in general significantly relaxed (see [14]), implies for example that a Liouville theorem applies to the radial weight functions $\varphi^{2}(x)=\left(1+\|x\|^{2}\right)^{\alpha}$, for any $\alpha \in \mathbb{R}$ (see [13] for more general results in this setting). On the other hand, it should be remarked that this is a quite heavy condition. For instance it does not apply to the case when "lower order terms" are constant.

In this paper a Liouville theorem is proved in the case of bounded weight functions $\varphi^{2}(x)$ which only depend on one or two variables, respectively $\varphi^{2}\left(x_{n}\right)$ and $\varphi^{2}\left(x_{n-1}, x_{n}\right)$ (see Sections 2 and 3 ). Thus we consider here the case of functions $\varphi^{2}(x)$ which satisfy symmetry assumptions different from the radial symmetry. For instance the weight function $\varphi^{2}\left(x_{n}\right):=\mathrm{e}^{-\sqrt{1+x_{n}^{2}}}$ does not satisfy the $\mathrm{O}\left(\left|x_{n}\right|^{-1}\right)$-decay condition on the "lower order term" $\frac{2 \varphi^{\prime}}{\varphi}$, moreover the integrability conditions on the weight function given in [16] do not hold either; yet a Liouville-type result can be proved in this setting (see Theorem 2.1).

Moreover making use of the technique of differential inequalities it is possible to state another type of Liouville theorem, replacing the boundedness condition on $\sigma$ by an assumption on the rate of divergence for its $L^{2}$ weighted integrals, where $\sigma$ now denotes more generally an arbitrary solution of the inequality $\sigma \nabla \cdot\left(\varphi^{2} \nabla \sigma\right) \geqslant 0$ in $\mathbb{R}^{n}$. In this direction in Section 5, we improve a Liouville-type result which played a key role in the proof of a conjecture of De Giorgi (see (3) [6, p. 175]): 
Conjecture [6]. Let us consider a solution $u \in C^{2}\left(\mathbb{R}^{n}\right)$ of

$$
\Delta u=u^{3}-u
$$

such that $|u| \leqslant 1, \partial_{n} u>0$ in the whole $\mathbb{R}^{n}$. Is it true that, for every $\lambda \in \mathbb{R}$, the sets $\{u=\lambda\}$ are hyperplanes, at least if $n \leqslant 8$ ?

Ghoussoub and Gui proved the conjecture in 1997 when $n=2$ (see [10] and [5]), Ambrosio and Cabré proved it in 2000 when $n$ is 3 (see [1]).

The proofs for $n=2$ and 3 use some techniques in the linear theory developed by Berestycki, Caffarelli and Nirenberg in [5].

That is, for every coordinate $x_{i}, i=1, \ldots, n-1$, consider the function $\sigma_{i}:=\partial_{i} u / \partial_{n} u$, where $\partial_{i} u=\partial u / \partial x_{i}$. The goal is to show that, for every $i, \sigma_{i}$ is constant (then $u$ is constant along $n-1$ directions, hence $u$ is a function of one variable only and the conjecture follows). This will be achieved using a Liouville-type result for the linear degenerate elliptic equation satisfied by $\sigma_{i}$, which turns out to be of the type we are interested in. Indeed using the fact that $\partial_{i} u$ and $\partial_{n} u$ satisfy the same linearized equation: $\Delta \omega-\left(3 u^{2}-1\right) \omega=0$, one can easily show that $\sigma_{i}$ satisfies: $\nabla \cdot\left(\varphi^{2} \nabla \sigma_{i}\right)=0$ in $\mathbb{R}^{n}$, where $\varphi:=\partial_{n} u$. This motivates the present work.

Some simple regularity results and bounds (see [1] for example) assure that $|\nabla u|$ is bounded in the whole $\mathbb{R}^{n}$, thus $\varphi \sigma_{i}$ is bounded in $\mathbb{R}^{n}$ (since by definition it equals to $\partial_{i} u$ ); hence the assumption of Berestycki, Caffarelli and Nirenberg Liouville theorem in [5] (which we recall in Remark 5.2) holds when $n=2$. This is essentially the proof of Ghoussoub and Gui (see [10] and [5]).

On the other hand the assumption in the Liouville theorem given in [1], which improves the one in [5] (we recall it in Remark 5.2, for the convenience of the reader), could be verified when (and only when) $n \leqslant 3$, due to the (optimal) energy estimate given in [1]; thus proving the conjecture when $n=3$.

Here in Theorem 5.1 we improve the Liouville-type results given in [1] and [5].

We notice that recently De Giorgi conjecture has been solved by Ovidiu Savin for $n \leqslant 8$, by means of a different approach (see [21]).

As a further motivation to the present work let us observe that the degenerate elliptic equations, whose weight functions decay exponentially along some direction, as we consider in Section 2, naturally arise in the proof of the one-dimensional character or symmetry of bounded monotone solutions to semilinear elliptic equations in the whole space $\mathbb{R}^{n}$. Indeed the explicit one-dimensional solution of Eq. (1.1) (which is unique up to translations of the independent variable) is given by $u(x)=\tanh \left(\frac{a \cdot x-c}{\sqrt{2}}\right)$ (for some $c \in \mathbb{R}$ and $a \in \mathbb{R}^{n}$ with $\|a\|=1$ and $a_{n}>0$ ); hence in order to prove the conjecture, following the above scheme, a useful tool would be a Liouville-type result which applies to a degenerate elliptic equation whose weight function $\varphi(x)$ could be $\partial_{n} u=\left(a_{n} / \sqrt{2}\right)\left(\cosh \left(\frac{a \cdot x-c}{\sqrt{2}}\right)\right)^{-2}$. This motivates the results in Section 2.

The structure of the paper is as follows. In Section 2 we prove a Liouville theorem concerning weight functions which only depend on one variable. Section 3 is devoted to the proof of a Liouville-type result for weight functions $\varphi^{2}$ which only depend on two variables. A new maximum principle for subsolutions is then proved in Section 4, under some assumptions on the weight $\varphi^{2}$, which cannot be essentially sharpened. It generalizes a result of [3], which by the end was proved through a probabilistic approach. As an application we show that the Liouville-type result, given in Section 2, applies to solutions $\sigma$ which are not a priori bounded. Finally in Section 5 we improve a Liouville-type result due to Berestycki, Caffarelli and Nirenberg. A classical counterexample shows that the asymptotic rate under which the Liouville theorem holds cannot be any more essentially sharpened when $n=2$; some counterexamples are given for $n \geqslant 9$ too.

In the following we usually set $\|x\|=\left(\sum_{i=1}^{n}\left(x_{i}\right)^{2}\right)^{1 / 2}$, for any $x \in \mathbb{R}^{n},\left|x_{n}\right|=\max \left\{-x_{n}, x_{n}\right\}, B_{R}=\{x \in$ $\left.\mathbb{R}^{n}:\|x\|<R\right\}$ and $\partial B_{R}=\left\{x \in \mathbb{R}^{n}:\|x\|=R\right\}$. 


\section{A Liouville theorem for one-dimensional weights}

Let us state the first Liouville-type result we obtained through a gradient estimate following from the maximum principle (see [19] for a general approach to the method).

Theorem 2.1. Let $\varphi$ be a positive $C^{1}$ function, $\varphi(x):=\varphi\left(x_{n}\right)$, such that:

(i) $\varphi \in L^{\infty}(\mathbb{R})$,

(ii) $\left(\varphi^{\prime}\left(x_{n}\right) \cdot x_{n}\right)^{+} \leqslant K \varphi\left(x_{n}\right)$ in $\mathbb{R}, \exists K \geqslant 0$.

Suppose that $\sigma \in L^{\infty}\left(\mathbb{R}^{n}\right) \cap C^{2}\left(\mathbb{R}^{n}\right)$ satisfies:

$$
\nabla \cdot\left(\varphi^{2}\left(x_{n}\right) \nabla \sigma\right)=0 \quad \text { in } \mathbb{R}^{n}
$$

in the classical sense, $\forall n \geqslant 1$. Then $\sigma$ is constant.

Theorem 2.1 holds for any $n \geqslant 1$ the interesting case, due to the above introduction, being the case $n \geqslant 3$.

Let us remark that under the above assumptions the operators under study are locally uniformly elliptic ones.

Remark 2.2. Let us notice that hypothesis (ii) in the theorem, when choosing $K=0$ in it, simply stands for a nonincreasing assumption on $\varphi$ as $\left|x_{n}\right|$ increases, with no asymptotic rate restriction. The possibility to choose a positive $K$ allows us to include the case of somewhere increasing weight functions.

Remark 2.3. Theorem 2.1 applies to equation $\nabla \cdot\left(\mathrm{e}^{-\alpha \sqrt{1+x_{n}^{2}}} \nabla \sigma\right)=0$ in $\mathbb{R}^{n}$, for any $\alpha>0$. Let us observe that the "lower order coefficient" $\frac{2 \varphi^{\prime}}{\varphi}$, corresponding to this choice of $\varphi\left(x_{n}\right):=\mathrm{e}^{-\frac{\alpha}{2} \sqrt{1+x_{n}^{2}}}$, does not decay to zero as $\left|x_{n}\right| \rightarrow+\infty$.

Proof of the Theorem 2.1. Let us define the following $n-1$ new functions:

$$
\psi_{i}(x)=\frac{c}{R^{2}} \sigma^{2}+\xi_{R}^{2}\left(\partial_{i} \sigma\right)^{2}, \quad \text { for any } i=1, \ldots, n-1,
$$

where $\partial_{i}=\frac{\partial}{\partial_{x_{i}}}, \xi_{R}(x):=\xi(\|x\| / R), \xi$ being a $C^{2}$ function in $[0,1]$ such that $0 \leqslant \xi \leqslant 1, \xi^{\prime} \leqslant 0$ and

$$
\xi(s)= \begin{cases}1 & s \in\left[0, \frac{1}{2}\right] \\ 0 & s \geqslant 1\end{cases}
$$

and $c$ is some constant in $\mathbb{R}^{+}$, that will be fixed later on.

Claim. $L \psi_{i} \geqslant 0$ in $\mathbb{R}^{n}$ where $L:=\frac{1}{\varphi^{2}} \nabla \cdot\left(\varphi^{2} \nabla\right)=\Delta+\frac{2}{\varphi} \varphi^{\prime} \partial_{n}$.

Due to the fact that $\varphi$ is positive in $\mathbb{R}$ and $\varphi^{\prime}$ is bounded in each compact set of $\mathbb{R}$, since $\varphi \in C^{1}(\mathbb{R})$, it follows that $\frac{\varphi^{\prime}\left(x_{n}\right)}{\varphi\left(x_{n}\right)} \in L_{\mathrm{loc}}^{\infty}(\mathbb{R})$. Thus a maximum principle on bounded domains holds for $L$ (see [12]), saying us that $\forall R>0$ :

$$
\max _{B_{R}} \psi_{i} \leqslant \max _{\partial B_{R}} \psi_{i}=\frac{c}{R^{2}} \max _{\partial B_{R}} \sigma^{2} \leqslant \frac{c}{R^{2}}\|\sigma\|_{L^{\infty}\left(\mathbb{R}^{n}\right)}^{2} .
$$

On the other hand:

$$
\max _{B_{R}} \psi_{i} \geqslant \max _{B_{\frac{R}{2}}} \psi_{i} \geqslant \max _{B_{\frac{R}{2}}}\left(\partial_{i} \sigma\right)^{2} \geqslant\left(\partial_{i} \sigma\right)^{2}(x), \quad \forall x \in B_{\frac{R}{2}} .
$$


From above, one can easily infer that:

$$
\left|\partial_{i} \sigma(x)\right| \leqslant \frac{\sqrt{c}}{R}\|\sigma\|_{L^{\infty}\left(\mathbb{R}^{n}\right)} \quad \forall x \in B_{\frac{R}{2}}
$$

letting $R \rightarrow+\infty$, it follows that $\left(\partial_{i} \sigma\right)(x)=0 \forall i=1, \ldots, n-1$. It implies that the function $\sigma$, which a priori depends on $n$ variables, indeed only depends on $x_{n}$, that is $\sigma\left(x_{1}, \ldots, x_{n}\right)=\sigma\left(x_{n}\right)$. This is a symmetry result.

Coming back to our equation we easily find out that now $\sigma$ solves the following one-dimensional problem:

$$
\partial_{n}\left(\varphi^{2}\left(x_{n}\right) \partial_{n} \sigma\right)=0 \quad \text { in } \mathbb{R}
$$

which equivalently implies:

$$
\partial_{n} \sigma=\frac{C}{\varphi^{2}\left(x_{n}\right)} \text { in } \mathbb{R}
$$

for some constant $C$. Integrating both sides of the previous inequality, we get:

$$
\sigma\left(x_{n}\right)=\int_{a}^{x_{n}} \frac{C}{\varphi^{2}(s)} \mathrm{d} s+\sigma(a),
$$

fixed arbitrarily $a \in \mathbb{R}$. Making use of hypothesis (i) it follows that the previous function $\sigma$ is bounded in $\mathbb{R}$ if and only if $C=0$; thus the only bounded solutions $\sigma$ of (2.1) are the constants. This proves the theorem.

Indeed $\int_{a}^{x_{n}} \varphi^{-2}(s) \mathrm{d} s \geqslant\left(\left\|\varphi^{2}\right\|_{L^{\infty}(\mathbb{R})}\right)^{-1}\left(x_{n}-a\right)$, which is obviously unbounded for any $a \in \mathbb{R}$.

Proof of the Claim. Let us calculate $L \psi_{i}$. Since $L \Phi^{2}=2\left(\Phi L \Phi+|\nabla \Phi|^{2}\right)$ it easily follows that:

$$
L \psi_{i}=\frac{c}{R^{2}} L \sigma^{2}+L\left(\left[\xi_{R} \partial_{i} \sigma\right]^{2}\right)=\frac{2 c}{R^{2}}|\nabla \sigma|^{2}+2\left(\xi_{R} \partial_{i} \sigma L\left(\xi_{R} \partial_{i} \sigma\right)+\left|\nabla\left(\xi_{R} \partial_{i} \sigma\right)\right|^{2}\right) .
$$

In any case:

$$
L\left(\xi_{R} \partial_{i} \sigma\right)=\xi_{R} L\left(\partial_{i} \sigma\right)+\left(\partial_{i} \sigma\right) L \xi_{R}+2 \nabla \xi_{R} \nabla \partial_{i} \sigma
$$

since $i=1, \ldots, n-1$, and $\varphi$ is a $x_{n}$-weight, from the fact that $L \sigma=0$ it is true that $L\left(\partial_{i} \sigma\right)=\partial_{i}(L(\sigma))=0$ in the weak sense in $\mathbb{R}^{n}$. From (2.2) it follows that:

$$
\begin{aligned}
L \psi_{i}= & \frac{2 c}{R^{2}}|\nabla \sigma|^{2}+2 \xi_{R}\left(\partial_{i} \sigma\right)^{2}\left(\Delta \xi_{R}+2 \frac{\varphi^{\prime}\left(x_{n}\right)}{\varphi\left(x_{n}\right)} \partial_{n} \xi_{R}\right)+4 \xi_{R} \partial_{i} \sigma \nabla \xi_{R} \nabla \partial_{i} \sigma \\
& +2\left(\left|\nabla \xi_{R}\right|^{2}\left(\partial_{i} \sigma\right)^{2}+\left|\nabla \partial_{i} \sigma\right|^{2} \xi_{R}^{2}+2 \xi_{R} \partial_{i} \sigma \nabla \xi_{R} \nabla \partial_{i} \sigma\right) \\
= & \frac{2 c}{R^{2}}|\nabla \sigma|^{2}+2 \xi_{R}\left(\partial_{i} \sigma\right)^{2} \Delta \xi_{R}+4 \xi_{R}\left(\partial_{i} \sigma\right)^{2} \frac{\varphi^{\prime}\left(x_{n}\right)}{\varphi\left(x_{n}\right)} \xi^{\prime} \frac{x_{n}}{R\|x\|} \\
& +8 \xi_{R} \partial_{i} \sigma \nabla \xi_{R} \nabla \partial_{i} \sigma+2\left|\nabla \xi_{R}\right|^{2}\left(\partial_{i} \sigma\right)^{2}+2\left|\nabla \partial_{i} \sigma\right|^{2} \xi_{R}^{2} \\
\geqslant & \frac{2 c}{R^{2}}|\nabla \sigma|^{2}+2 \xi_{R}\left(\partial_{i} \sigma\right)^{2} \Delta \xi_{R}-4 \xi_{R}\left|\xi^{\prime}\right| \frac{\left(\varphi^{\prime}\left(x_{n}\right) \cdot x_{n}\right)^{+}}{\varphi\left(x_{n}\right)} \frac{\left(\partial_{i} \sigma\right)^{2}}{R\|x\|}+8 \xi_{R} \partial_{i} \sigma \nabla \xi_{R} \nabla \partial_{i} \sigma+2\left|\nabla \partial_{i} \sigma\right|^{2} \xi_{R}^{2} .
\end{aligned}
$$

Making use of hypothesis (ii), since $\xi_{R}^{\prime}$ vanishes in $B_{\frac{R}{2}}$, one can in the last term estimate $\frac{1}{\|x\|}$ from above by $\frac{2}{R}$; thus:

$$
L \psi_{i} \geqslant\left(2 c-c_{1}-c_{2} K-\frac{c_{3}}{\varepsilon^{2}}\right) \frac{\left(\partial_{i} \sigma\right)^{2}}{R^{2}}+\left(2-(8 \varepsilon)^{2}\right)\left|\nabla \partial_{i} \sigma\right|^{2} \xi_{R}^{2}
$$

where $c_{1}, c_{2}, c_{3}$ are constants that only depends on $\xi$ (and its derivatives up to the second order), and we make use of Young inequality in the third term. Choosing $\varepsilon$ small and $c$ big enough, the Claim follows from the nonnegativity of the right hand side. 
Remark 2.4. Theorem 2.1 more generally applies to weight functions $\varphi^{2}$ which instead of being bounded are such that $\varphi^{-1} \notin L^{2}((a,+\infty)), \forall a \in \mathbb{R}$; this is clear from the proof.

Remark 2.5. If condition (ii) holds only on $\left\{\left|x_{n}\right| \gg 1\right\}$ the claim easily follows making use of a cylindrical, instead of the previous radial, $\xi_{R}$ (at least for $R$ big enough):

$$
\xi_{R}(x):=\xi\left(\frac{\left|x_{n}\right|}{R}\right) \xi\left(\frac{\left\|x^{\prime}\right\|}{R}\right)
$$

where $\xi$ is the same as before, and we denote $x=\left(x^{\prime}, x_{n}\right) \in \mathbb{R}^{n-1} \times \mathbb{R},\left\|x^{\prime}\right\|=\left(\sum_{i=1}^{n-1}\left(x_{i}\right)^{2}\right)^{1 / 2}$.

\section{A Liouville theorem for two-dimensional weights}

Arguing as in Theorem 2.1 a second Liouville-type result can be stated.

Theorem 3.1. Let $\varphi$ be a positive $C^{1}$ function, $\varphi(x):=\varphi\left(x_{n-1}, x_{n}\right)$, such that:

(i) $\varphi \in L^{\infty}\left(\mathbb{R}^{2}\right)$,

(ii) $\left(\partial_{n-1} \varphi \cdot x_{n-1}+\partial_{n} \varphi \cdot x_{n}\right)^{+} \leqslant K \varphi\left(x_{n-1}, x_{n}\right)$ in $\mathbb{R}^{2}, \exists K \geqslant 0$.

Suppose that $\sigma \in L^{\infty}\left(\mathbb{R}^{n}\right) \cap C^{2}\left(\mathbb{R}^{n}\right)$ satisfies:

$$
\nabla \cdot\left(\varphi^{2}\left(x_{n-1}, x_{n}\right) \nabla \sigma\right)=0 \quad \text { in } \mathbb{R}^{n}
$$

in the classical sense, $\forall n \geqslant 2$. Then $\sigma$ is constant.

Once again Theorem 3.1 holds for any $n \geqslant 2$ the interesting case, due to the above introduction, being the case $n \geqslant 3$.

Its proof goes as the one of Theorem 2.1. Making use only of the first $n-2$ functions $\psi_{i}, i=1, \ldots, n-2$ (which are defined exactly as in the proof of Theorem 2.1), one can now easily show that $\sigma$, which a priori depends on $n$ variables, indeed only depends on $x_{n-1}$ and $x_{n}$, that is $\sigma\left(x_{1}, \ldots, x_{n}\right)=\sigma\left(x_{n-1}, x_{n}\right)$. This is a symmetry result. Thus $\sigma$ is a bounded solution of the following two-dimensional problem

$$
\sum_{i=n-1}^{n} \frac{\partial}{\partial_{x_{i}}}\left(\varphi^{2}\left(x_{n-1}, x_{n}\right) \frac{\partial \sigma}{\partial_{x_{i}}}\right)=0 \text { in } \mathbb{R}^{2} .
$$

Since $\varphi^{2}$ is by assumption positive and bounded, Finn Liouville-type result (see [9,11]) states that $\sigma$ must be constant; this proves the theorem.

Remark 3.2. Let us notice that hypothesis (ii), when choosing $K=0$ in it, simply stands for a nonincreasing assumption on $\varphi$ as the "radius" $\sqrt{\left(x_{n-1}\right)^{2}+\left(x_{n}\right)^{2}}$ increases, with no asymptotic rate restriction.

Remark 3.3. It should be notice that the preceding proof cannot be straightforward generalized for example to three-dimensional weight functions $\varphi^{2}\left(x_{n-2}, x_{n-1}, x_{n}\right)$. This is due to the fact that a Liouville theorem in dimension $n=3$ is not true under the sole boundedness assumption on the positive function $\varphi^{2}$ (see [2]).

\section{A maximum principle in unbounded domains}

In this section we prove a new version of the maximum principle on unbounded domains for the degenerate elliptic operators in divergence form under study. It makes use of a result of the same kind (but for the uniformly 
elliptic case) contained in one of Berestycki, Caffarelli and Nirenberg's papers, concerned with the study of qualitative properties of semilinear elliptic equations in various type of unbounded domains.

Let us first remind that result, denoted by Lemma 2.1 in [4]:

Theorem 4.1. Let $D$ be a domain (open connected set) in $\mathbb{R}^{n}$, possibly unbounded. Assume that $\bar{D}$ is disjoint from the closure of an infinite open connected cone. Suppose there is a function $z$ in $C(\bar{D})$ that is bounded above and satisfies for some continuous function $c(x) \leqslant 0$ :

$$
\begin{cases}\Delta z+c(x) z \geqslant 0 & \text { in } D \\ z \leqslant 0 & \text { on } \partial D .\end{cases}
$$

Then $z \leqslant 0$ in $D$.

We suppose that $z \in W_{\text {loc }}^{2, n}(D)$. Let us remark that the same result holds if $c(x)$ is a measurable, not necessarily continuous function in $D$, as in the Alexandroff-Bakelman-Pucci estimate.

For the degenerate elliptic case the following holds:

Theorem 4.2. Let $D$ be a domain (open connected set) in $\mathbb{R}^{n}$, possibly unbounded. Assume that $\bar{D}$ is disjoint from the closure of an infinite open connected cone. Let $\varphi$ be a positive function in $D, \varphi \in W_{\mathrm{loc}}^{2, n}(D)$, that satisfies the following:

(i) $\varphi \in L^{\infty}(D)$,

(ii) $\Delta \varphi \geqslant 0$ a.e. in $D$.

Suppose there is a function $\sigma$, such that $\varphi \sigma \in C(\bar{D})$ and is bounded above in D. Suppose moreover that $\sigma \in C^{2}(D)$ satisfies:

$$
\begin{cases}-\nabla \cdot\left(\varphi^{2} \nabla \sigma\right) \leqslant 0 & \text { in } D \\ \sigma \leqslant 0 & \text { on } \partial D .\end{cases}
$$

Then $\sigma \leqslant 0$ in $D$.

Proof. Let us consider in $D$ the auxiliary function

$$
\omega(x)=\varphi^{-1}(x) .
$$

Since $\varphi$ is positive and bounded in $D$, the function $\omega$ is strictly positive in $D$. Moreover the function:

$$
h(x)=\frac{\sigma(x)}{\omega(x)}
$$

satisfies in $D$ the following problem:

$$
\begin{cases}\Delta h-\frac{\Delta \varphi}{\varphi} h \geqslant 0 & \text { in } D, \\ h \leqslant 0 & \text { on } \partial D .\end{cases}
$$

Thanks to the hypothesis made in the theorem, it is true that $c(x):=\frac{-\Delta \varphi}{\varphi}$ is a measurable nonpositive function a.e. in $D$, and that the function $h=\varphi \sigma \in C(\bar{D}) \cap W_{\text {loc }}^{2, n}(D)$, is bounded from above in $D$. From this we infer, by Theorem 4.1, that $h \leqslant 0$ in $D$. Hence $\sigma \leqslant 0$ in $D$.

Corollary 4.3. Under the same hypothesis on $\varphi$ and $D$, suppose there is a function $\sigma$, such that $\varphi \sigma \in C(\bar{D})$ and is bounded below in D. Suppose moreover that $\sigma \in C^{2}(D)$ satisfies:

$$
\begin{cases}-\nabla \cdot\left(\varphi^{2} \nabla \sigma\right) \geqslant 0 & \text { in } D \\ \sigma \geqslant 0 & \text { on } \partial D .\end{cases}
$$


Then $\sigma \geqslant 0$ in $D$.

Proof. It suffices to apply Theorem 4.2 to $-\sigma$.

Let us note at the end that from the previous results one can easily prove now the following, already proved in [3] (see Proposition 2.7), by means of a probabilistic approach and under stronger assumptions on the weight $\varphi$.

Theorem 4.4. Let $\varphi$ be a positive function in $D, \varphi \in W_{\mathrm{loc}}^{2, n}(D)$. Suppose that:

(i) $\varphi \geqslant \varepsilon_{0}>0$ a.e. in $\mathbb{R}^{n} \backslash D$,

(ii) $\varphi \in L^{\infty}(D)$,

(iii) $\Delta \varphi \geqslant 0$ a.e. in $D$;

where $D$ is any domain (open connected set) in $\mathbb{R}^{n}$, possibly unbounded, such that $\bar{D}$ is disjoint from the closure of an infinite open connected cone. If $\sigma \in C^{2}(D)$ is a solution of $\nabla \cdot\left(\varphi^{2} \nabla \sigma\right)=0$ in $\mathbb{R}^{n}$ such that $\varphi \sigma \in L^{\infty}\left(\mathbb{R}^{n}\right) \cap$ $C(\bar{D})$ then $\sigma \in L^{\infty}\left(\mathbb{R}^{n}\right)$.

Proof. Since by hypothesis $\varphi \geqslant \varepsilon_{0}>0$ a.e. outside $D$, it easily follows that $\sigma \in L^{\infty}\left(\mathbb{R}^{n} \backslash D\right)$. From this, making use of Theorem 4.2 and Corollary 4.3, the claim easily follows.

Remark 4.5. The hypothesis made in Theorem 4.2 are in some sense sharp, as it is shown by the examples following:

Example 1. Let us in fact consider the function $\sigma\left(x^{\prime}, x_{n}\right)=\mathrm{e}^{-x_{n}}$ on the domain $D=\left\{x \in \mathbb{R}^{n}: x_{n} \geqslant 1\right\}$. On this domain the function can be easily seen to satisfy, in the classical sense, the following problem:

$$
\nabla \cdot\left(\mathrm{e}^{x_{n}} \nabla \sigma\right)=0 .
$$

In any case:

$$
0=\inf _{D} \sigma<\inf _{\partial D} \sigma=\mathrm{e}^{-1} .
$$

One can easily verify that in this case $\varphi\left(x_{n}\right)=\mathrm{e}^{x_{n} / 2}$ does not belong to $L^{\infty}(D)$. On the contrary all the remaining hypotheses, made in Corollary 4.3, on $\sigma$ and $\varphi$ are satisfied (see Fig. 1).

The previous example shows the sharpness of the boundedness (from above) condition on $\varphi$, on the other hand the following one shows the sharpness of the one side boundedness condition on $\varphi \sigma$ over the set $D$.
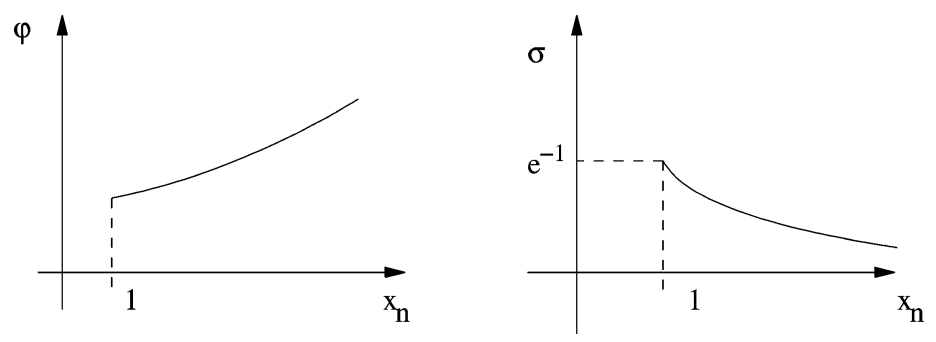

Fig. 1. $\varphi \notin L^{\infty}(D)$. 

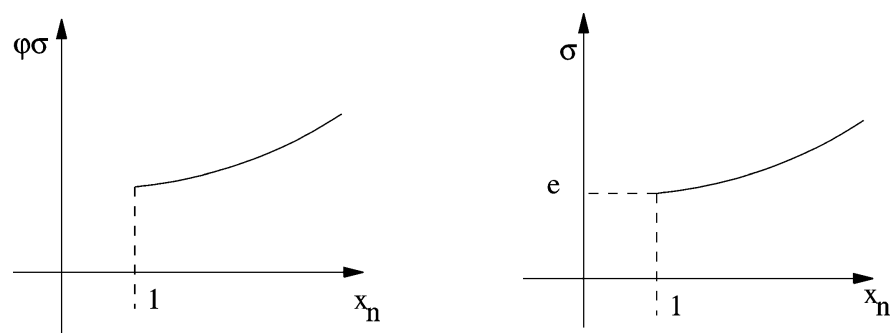

Fig. 2. $\varphi \sigma \notin L^{\infty}(D)$.

Example 2. Let us consider now the function $\sigma\left(x^{\prime}, x_{n}\right)=\mathrm{e}^{x_{n}}$ on the same domain $D=\left\{x \in \mathbb{R}^{n}: x_{n} \geqslant 1\right\}$. On this domain the function can be easily seen to satisfy, in the classical sense, the following problem:

$$
\nabla \cdot\left(\mathrm{e}^{-x_{n}} \nabla \sigma\right)=0 .
$$

In any case:

$$
+\infty=\sup _{D} \sigma>\sup _{\partial D} \sigma=\mathrm{e} .
$$

One can easily verify that in this case $\varphi\left(x_{n}\right)=\mathrm{e}^{-x_{n} / 2}$ so that $\varphi \sigma=\mathrm{e}^{x_{n} / 2}$ is not bounded above in $D$. On the contrary once again all the remaining hypotheses, included in Theorem 4.2, on $\sigma$ and $\varphi$ are satisfied (see Fig. 2).

An easy application of the above maximum principle leads us to improve in some cases Theorem 2.1 as follows.

Theorem 4.6. Let $\varphi(x):=\varphi\left(x_{n}\right) \in C^{2}(\mathbb{R})$ be a positive function such that:

(i) $\varphi \in L^{\infty}(\mathbb{R})$,

(ii) $\varphi^{\prime \prime}\left(x_{n}\right) \geqslant 0$ for $\left|x_{n}\right|$ big enough,

(iii) $\lim _{x_{n} \rightarrow \pm \infty} \varphi^{\prime}\left(x_{n}\right)=0$.

Suppose that $\varphi \sigma \in L^{\infty}\left(\mathbb{R}^{n}\right)$ and $\sigma \in C^{2}\left(\mathbb{R}^{n}\right)$ satisfies:

$$
\nabla \cdot\left(\varphi^{2}\left(x_{n}\right) \nabla \sigma\right)=0 \quad \text { in } \mathbb{R}^{n}
$$

in the classical sense, $\forall n \geqslant 1$. Then $\sigma$ is constant.

Proof. As in Theorem 4.4 one can prove that $\sigma \in L^{\infty}\left(\mathbb{R}^{n} \backslash D\right)$ where $D=D_{1} \cup D_{2}$ and $D_{1}=\left\{x_{n} \gg 1\right\}, D_{2}=$ $\left\{x_{n} \ll-1\right\}$. Making use twice of Theorem 4.2 and Corollary 4.3 (once in $D_{1}$ and then in $D_{2}$ ), it follows that $\sigma \in L^{\infty}\left(\mathbb{R}^{n}\right)$. To this end condition (ii) is needed. On the other hand assumptions (ii) and (iii) here implies that in $D, \varphi$ does not increase as $\left|x_{n}\right|$ increases. Due to Remarks 2.2 and 2.5, from Theorem 2.1 the thesis follows.

Remark 4.7. The above theorem implies that not only every bounded solution $\sigma(x)$ of $\nabla \cdot\left(\mathrm{e}^{-2 \sqrt{1+x_{n}^{2}}} \nabla \sigma\right)=0$ in $\mathbb{R}^{n}$, must be constant, to this end Theorem 2.1 will suffice (see Remark 2.3); yet the same holds true for every solution $\sigma(x)$ which diverge at most like $\mathrm{e}^{\left|x_{n}\right|}$ as $\left|x_{n}\right| \rightarrow \infty$, still being bounded in the first $n-1$ variables.

\section{An improvement of a Berestycki-Caffarelli-Nirenberg Liouville theorem}

Here we deal with the asymptotic behavior of solutions of the corresponding second-order partial differential inequalities in $n$ independent variables. 
To conveniently state our result, let us set

$$
\mathcal{F}=\left\{F: \mathbb{R}^{+} \rightarrow \mathbb{R}^{+}, F \text { is nondecreasing and } \sum_{j=0}^{\infty} \frac{1}{F\left(2^{j+1}\right)}=+\infty\right\} .
$$

Thus $\mathcal{F}$ includes such functions as $\log (1+r), \log (1+r) \cdot \log (1+\log (1+r))$.

Theorem 5.1. Let $\varphi \in L_{\mathrm{loc}}^{\infty}\left(\mathbb{R}^{n}\right)$ be a positive function. Suppose that $\sigma \in H_{\mathrm{loc}}^{1}\left(\mathbb{R}^{n}\right)$ satisfies

$$
\sigma \nabla \cdot\left(\varphi^{2} \nabla \sigma\right) \geqslant 0 \quad \text { in } \mathbb{R}^{n}
$$

in the distributional sense, $n \geqslant 1$. Let this condition holds:

$$
\limsup _{R \rightarrow+\infty} \frac{1}{R^{2} F(R)} \int_{B_{R}}(\varphi \sigma)^{2} \mathrm{~d} x=C
$$

for some finite constant $C$, and some $F \in \mathcal{F}$. Then $\sigma$ is constant.

Remark 5.2. When choosing $F(R) \equiv 1$ in (5.2) we recover Proposition 2.1 of [1], which generalizes for $n \geqslant 3$, the decay assumption on $\varphi \sigma$ made in Berestycki, Caffarelli and Nirenberg Liouville property (see Theorem 1.8 in [5]), showing that it is possible to carry out their proof even under this less restrictive hypothesis. Let us recall one of its consequences: any functions $\sigma$ such that $\varphi \sigma \in L^{\infty}\left(\mathbb{R}^{n}\right)$, satisfying the differential inequality (5.1) when $n=2$ is indeed constant (since the Euclidean measure of a ball of radius $R$ goes like $R^{2}$, in the Euclidean plane). Thus in particular Berestycki, Caffarelli and Nirenberg Liouville property recovers Finn Liouville-type result (see [9,11]).

Corollary 5.3. Let $\varphi \in L_{\mathrm{loc}}^{\infty}\left(\mathbb{R}^{n}\right)$ be a positive function. Suppose that $\sigma \in H_{\mathrm{loc}}^{1}\left(\mathbb{R}^{n}\right)$ satisfies

$$
\sigma \nabla \cdot\left(\varphi^{2} \nabla \sigma\right) \geqslant 0 \quad \text { in } \mathbb{R}^{n}
$$

in the distributional sense, $n \geqslant 1$. For every $R>1$, assume that:

$$
\int_{B_{R}}(\varphi \sigma)^{2} \mathrm{~d} x \leqslant C R^{2} \log R
$$

for some constant $C$ independent of $R$. Then $\sigma$ is constant.

The proofs of the above results make use of the method of differential inequalities (see [15] for a general approach to the method); hence they are different from the proof of Berestycki, Caffarelli and Nirenberg Liouville property, given in [5], which seems it cannot be improved to cover the present setting.

Remark 5.4. We discuss here a classical example (see [20]) bearing on the sharpness of the preceding result when $n=2$.

Let $\varphi=1$. There exists at least a nonconstant $\sigma$ such that: $\sigma \cdot \Delta \sigma \geqslant 0$ in $\mathbb{R}^{2}$, in the classical sense, for which

$$
\limsup _{R \rightarrow+\infty} \frac{1}{R^{2}(\log R)^{2}} \int_{B_{R}} \sigma^{2} \mathrm{~d} x=C
$$

for some finite constant $C$. Simply take

$$
\sigma(x)= \begin{cases}\left(\log R_{0}-\frac{3}{4}\right)+\frac{r^{2}}{R_{0}{ }^{2}}-\frac{r^{4}}{4 R_{0}{ }^{4}} & r<R_{0}, \\ \log r & r \geqslant R_{0},\end{cases}
$$


where $r$ denotes as usual $\|x\|$, and $R_{0}$ is chosen: $R_{0}>\mathrm{e}^{3 / 4}$.

This shows that the previous theorem cannot be, in general appreciably improved when $n=2$. It holds for $R^{2} \log R$, while it does not for $R^{2}(\log R)^{2}$. On the other hand, in some special cases these rates can certainly be improved.

Remark 5.5. An interesting open problem is either to prove or to give a counterexample to Theorem 5.1 when $F(R)=R^{n-3}$, thus $R^{2} F(R)=R^{n-1}$, and $4 \leqslant n \leqslant 8$; to this respect see [1].

When $n \geqslant 9$ a counterexample for this choice of $F$ can be easily obtained as in [10]. We give here only a sketch of its proof since it goes exactly as the one of Proposition 2.6 of [10].

Counterexample. Consider the bounded sign changing function $u(x):=\left(1+\|x\|^{2}\right)^{-\alpha} x_{1}$ with $\alpha:=n / 4-$ $(\sqrt{n-1}) / 2, n \geqslant 7$. The goal is to show that $\lambda_{1}\left(\frac{-\Delta u}{u}\right)=0$, where we denote by $\lambda_{1}\left(\frac{-\Delta u}{u}\right):=\inf \left\{\left(\int_{\mathbb{R}^{n}}|\nabla \eta|^{2}+\right.\right.$ $\left.\left.\frac{\Delta u}{u}|\eta|^{2} \mathrm{~d} x\right) /\left(\int_{\mathbb{R}^{n}}|\eta|^{2} \mathrm{~d} x\right)\right\} ; \eta \in C_{0}^{\infty}\left(\mathbb{R}^{n}\right)$. Indeed by means of the connection between Liouville property for operator $\nabla \cdot\left(\varphi^{2} \nabla \sigma\right)$ and the spectrum of linear Schrödinger operators (see [5], see also [10]) this implies that there exist a nonconstant function $\sigma$ and a positive function $\varphi$ such that $\nabla \cdot\left(\varphi^{2} \nabla \sigma\right)=0$ in $\mathbb{R}^{n}$ and $\varphi \sigma=u$, thus $\int_{B_{R}}(\varphi \sigma)^{2} \mathrm{~d} x=\int_{B_{R}} u^{2} \mathrm{~d} x \leqslant C R^{2+2 \sqrt{n-1}}$. Since $2+2 \sqrt{n-1} \leqslant n-1$ if and only if $n \geqslant 9$, the claim is proved.

In order to show that $\lambda_{1}\left(\frac{-\Delta u}{u}\right)=0$, we consider the positive bounded function $\psi(x):=\left(1+\|x\|^{2}\right)^{-\beta}$, where $\beta:=\frac{n-2}{4}$. By Proposition 2.3 and Lemma 2.1 in [10], $\lambda_{1}\left(\frac{-\Delta \psi}{\psi}\right)=0$ and $\lambda_{1}\left(\frac{-\Delta u}{u}\right) \leqslant 0$. Since for $n \geqslant 5$ we have $-\frac{\Delta u}{u} \leqslant-\frac{\Delta \psi}{\psi}$, it follows that: $0=\lambda_{1}\left(\frac{-\Delta \psi}{\psi}\right) \leqslant \lambda_{1}\left(\frac{-\Delta u}{u}\right) \leqslant 0$; thus the claim is proved.

Remark 5.6. Let us recall on the other hand that a counterexample to Theorem 5.1 when $F(R)=R^{n-2}$, thus $R^{2} F(R)=R^{n}$, can be found in [2] for any $n \geqslant 3$. When $n \geqslant 7$ a different counterexample to this case was previously given explicitly by Ghoussoub and Gui in [10] (see Proposition 2.8).

Proof of the Corollary 5.3. Let us choose $F(r)=\log r$ for $r>1$, such an $F$ indeed belongs to $\mathcal{F}$, then the claim easily follows from the previous theorem.

Proof of the Theorem 5.1. Let $\sigma$ satisfies $\sigma \nabla \cdot\left(\varphi^{2} \nabla \sigma\right) \geqslant 0$, we then have

$$
\nabla \cdot\left(\sigma \varphi^{2} \nabla \sigma\right)=\varphi^{2}|\nabla \sigma|^{2}+\sigma \nabla \cdot\left(\varphi^{2} \nabla \sigma\right) \geqslant \varphi^{2}|\nabla \sigma|^{2} .
$$

On the other hand,

$$
\int_{\partial B_{R}} \sigma \varphi^{2}(\nabla \sigma, \nu) \mathrm{d} s \leqslant \int_{\partial B_{R}}|\sigma| \varphi^{2}|\nabla \sigma| \mathrm{d} s \leqslant\left(\int_{\partial B_{R}} \varphi^{2}|\nabla \sigma|^{2} \mathrm{~d} s\right)^{\frac{1}{2}}\left(\int_{\partial B_{R}}(\varphi \sigma)^{2} \mathrm{~d} s\right)^{\frac{1}{2}},
$$

where $v$ denotes the outer unit normal vector on $\partial B_{R}$. Now let

$$
D(R)=\int_{B_{R}} \varphi^{2}|\nabla \sigma|^{2} \mathrm{~d} x .
$$

Integrating (5.3) over $B_{R}$ and using (5.4), we then have

$$
D(R) \leqslant D^{\prime}(R)^{\frac{1}{2}}\left(\int_{\partial B_{R}}(\varphi \sigma)^{2} \mathrm{~d} s\right)^{\frac{1}{2}} .
$$


If $\sigma \neq$ constant, then there exists some $R_{0}>0$ such that $D(R)>0$ for all $R \geqslant R_{0}$ and we may deduce from (5.5) that

$$
\frac{1}{D\left(r_{1}\right)}-\frac{1}{D\left(r_{2}\right)}=-\int_{r_{1}}^{r_{2}} \frac{\mathrm{d}}{\mathrm{d} R}\left(\frac{1}{D(R)}\right) \mathrm{d} R \geqslant\left(\int_{r_{1}}^{r_{2}}\left(\int_{\partial B_{R}}(\varphi \sigma)^{2} \mathrm{~d} s\right)^{-1} \mathrm{~d} R\right)
$$

for all $r_{2}>r_{1}>R_{0}$.

Claim. There holds:

$$
\left(r_{2}-r_{1}\right)^{2}\left(\int_{r_{1}}^{r_{2}}\left(\int_{\partial B_{R}}(\varphi \sigma)^{2} \mathrm{~d} s\right) \mathrm{d} R\right)^{-1} \leqslant\left(\int_{r_{1}}^{r_{2}}\left(\int_{\partial B_{R}}(\varphi \sigma)^{2} \mathrm{~d} s\right)^{-1} \mathrm{~d} R\right) .
$$

Proof of the Claim. By means of Schwarz inequality for any $f: \mathbb{R} \rightarrow \mathbb{R}$ one has:

$$
\left(\int_{r_{1}}^{r_{2}} 1 \mathrm{~d} R\right)^{2}=\left(\int_{r_{1}}^{r_{2}} f(R)^{\frac{1}{2}} f(R)^{-\frac{1}{2}} \mathrm{~d} R\right)^{2} \leqslant\left(\int_{r_{1}}^{r_{2}} f(R)^{-1} \mathrm{~d} R\right)\left(\int_{r_{1}}^{r_{2}} f(R) \mathrm{d} R\right)
$$

Then from (5.7), the Claim easily follows, choosing $f(R)=\int_{\partial B_{R}}(\varphi \sigma)^{2} \mathrm{~d} s$.

It follows from (5.6), by means of the claim that

$$
\left(r_{2}-r_{1}\right)^{2}\left(\int_{B_{r_{2}} \backslash B_{r_{1}}}(\varphi \sigma)^{2} \mathrm{~d} x\right)^{-1} \leqslant \frac{1}{D\left(r_{1}\right)}-\frac{1}{D\left(r_{2}\right)} .
$$

Now let $r_{2}=2^{j+1} r^{*}$ and $r_{1}=2^{j} r^{*}$ for some $r^{*}>R_{0}$, for each $0 \leqslant j \leqslant N-1$, from (5.8) it follows easily that

$$
\frac{\left(2^{j+1} r^{*}\right)^{2}}{4}\left(\int_{B_{2^{j+1} r^{*}}}(\varphi \sigma)^{2} \mathrm{~d} x\right)^{-1} \leqslant\left(2^{j} r^{*}\right)^{2}\left(\int_{B_{2^{j+1} r^{*}} \backslash B_{2^{j} r^{*}}}(\varphi \sigma)^{2} \mathrm{~d} x\right)^{-1} \leqslant \frac{1}{D\left(2^{j} r^{*}\right)}-\frac{1}{D\left(2^{j+1} r^{*}\right)},
$$

summing over $j$ we find, taking into account hypothesis (5.2)

$$
\frac{1}{D\left(r^{*}\right)} \geqslant \frac{1}{D\left(r^{*}\right)}-\frac{1}{D\left(2^{N} r^{*}\right)} \geqslant \frac{1}{4 C} \sum_{j=0}^{N-1} \frac{1}{F\left(2^{j+1} r^{*}\right)} .
$$

Since $F$ belongs to $\mathcal{F}$, it is true that $F\left(2^{j+1} r^{*}\right) \leqslant F\left(2^{j+j_{0}+1}\right)$ if $j_{0}$ is such that $r^{*} \leqslant 2^{j_{0}}$, thus the sum in (5.9) diverges as $N \rightarrow+\infty$ and this implies that $D\left(r^{*}\right)=0$ for all $r^{*}>R_{0}$; thus $\sigma$ is constant.

Remark 5.7. The key point is to study for such a degenerate elliptic inequality the question of the divergence of $\int_{B_{R}}(\varphi \sigma)^{2} \mathrm{~d} x$ instead of that of $\int_{B_{R}} \sigma^{2} \mathrm{~d} x$, as was done on the contrary in Theorem C of [15]. The feature that makes possible the treatment of degenerate elliptic inequalities by this method is the use, initiated by Finn of integrals $D(R)$ based on the actual quadratic form defined by $\varphi^{2}$, rather then Dirichlet type integrals, as is customary when dealing with uniformly elliptic ones (see [9]). The same kind of integral of a solution $\sigma$, as the one in the hypothesis, was considered in [5]. 


\section{Acknowledgements}

The author would like to thank Professor Xavier Cabré for his constant encouragement and valuable suggestions. The author is grateful to the Departament de Matemàtica Aplicada 1, Universitat Politècnica de Catalunya, Barcelona, for its hospitality during the academic year 1999/2000 when part of this work was carried out.

\section{References}

[1] L. Ambrosio, X. Cabré, Entire solutions of semilinear elliptic equations in $\mathbb{R}^{3}$ and a conjecture of De Giorgi, J. Amer. Math. Soc. 13 (4) (2000) $725-739$.

[2] M.T. Barlow, On the Liouville property for divergence form operators, Canad. J. Math. 50 (3) (1998) 487-496.

[3] M.T. Barlow, R.F. Bass, C. Gui, The Liouville property and a conjecture of De Giorgi, CPAM LIII (2000) 1007-1038.

[4] H. Berestycki, L. Caffarelli, L. Nirenberg, Monotonicity for elliptic equations in unbounded Lipschitz domains, CPAM L (1997) 10891111.

[5] H. Berestycki, L. Caffarelli, L. Nirenberg, Further qualitative properties for elliptic equations in unbounded domains, Ann. Scuola Norm. Sup. Pisa Cl. Sci. (4) 25 (1997) 69-94.

[6] E. De Giorgi, Convergence problems for functionals and operators, in: Proc. Int. Meeting on Recent Methods in Nonlinear Analysis (Rome, 1978), Pitagora, Bologna, 1979, pp. 131-188.

[7] E. De Giorgi, Sulla differenziabilitá e analiticitá delle estremali degli integrali multipli regolari, Mem. Accad. Sci. Torino Cl. Sci. 3 (1957) $25-43$.

[8] D.E. Edmunds, L.A. Peletier, A Liouville theorem for degenerate elliptic equations, J. London Math. Soc. (2) 7 (1973) 95-100.

[9] R. Finn, Sur quelques généralisations du théorème de Picard, C. R. Acad. Sci. Paris Sér. A-B 235 (1952) 596-598.

[10] N. Ghoussoub, C. Gui, On a conjecture of De Giorgi and some related problems, Math. Ann. 311 (1998) 481-491.

[11] D. Gilbarg, J. Serrin, On isolated singularities of solutions of second order elliptic differential equations, J. Analyse Math. 4 (1955-1956) 309-340.

[12] D. Gilbarg, N.S. Trudinger, Elliptic Partial Differential Equations of Second Order, second ed., Springer-Verlag, 1983.

[13] A. Grigor'yan, L. Saloff-Coste, Stability results for Harnack inequalities, preprint.

[14] L. Karp, Asymptotic behavior of solutions of elliptic equations I: Liouville-type theorems for linear and nonlinear equations on $\mathbb{R}^{n}$, J. Analyse Math. 39 (1981) 75-102.

[15] L. Karp, Asymptotic behavior of solutions of elliptic equations II: Analogues of Liouville's theorem for solutions of inequalities on $\mathbb{R}^{n}, n \geqslant 3$, J. Analyse Math. 39 (1981) 103-115.

[16] S.N. Kruzkov, Certain properties of solutions to elliptic equations, Soviet. Math. Dokl. 4 (1963) 686-695.

[17] J. Moser, On Harnack's theorem for elliptic differential equations, CPAM 14 (1961) 577-591.

[18] J. Nash, Continuity of solutions of parabolic and elliptic equations, Amer. J. Math. 80 (1958) 931-954.

[19] L.A. Peletier, J. Serrin, Gradient bounds and Liouville theorems for quasilinear elliptic equations, Ann. Scuola Norm. Sup. Pisa Cl. Sci. (4) 5 (1978) 65-104.

[20] M.H. Protter, H.F. Weinberger, Maximum Principles in Differential Equations, Prentice-Hall, Englewood Cliffs, NJ, 1967.

[21] O. Savin, Phase transitions: regularity of flat level sets, preprint.

[22] N.S. Trudinger, On the regularity of generalized solutions of linear, non-uniformly elliptic equations, Arch. Rat. Mech. Anal. 42 (1971) $51-62$. 